\title{
Effective calculation of laser stripping via a broad shape resonance
}

\author{
T. Gorlov and V. Danilov \\ Spallation Neutron Source, Oak Ridge National Laboratory, Oak Ridge, Tennessee 37830, USA
}

(Received 15 April 2010; published 23 July 2010)

\begin{abstract}
This paper presents the theory and method of calculation of laser assisted charge exchange injection via a broad shape resonance of the hydrogen atom with a new level of accuracy. The method is optimized for fast calculations, needed for practical estimations of laser-stripping efficiency for charge exchange injection. The novelty is that we take into account the resonance width and continuous spectrum of the hydrogen atom in our model. As a result we show that the broad shape resonance can fully compensate the Doppler broadening of the laser frequency in realistic beams without applying laser chirp and increasing laser power. The resulting scheme can be realized by applying a magnetic field of optimal strength to the high-energy beam irradiated by laser field. Another novelty is that we use the temporal Schrödinger equation as the basis of our model in contrast with the existing method of semiempirical cross sections, which is widely considered in atomic physics. The strict quantum mechanical approach gives the temporal evolution of the wave function and the ionization probability of the hydrogen atom as a function of laser and static electric fields. Moreover, it reveals quantum effects at the strong laser field which cannot be described with the cross section treatment. It is shown that the effects play a significant role in the optimization of the magnetic field for the laser-stripping scheme.
\end{abstract}

DOI: 10.1103/PhysRevSTAB.13.074002

PACS numbers: $32.80 . \mathrm{Fb}, 32.80 . \mathrm{Rm}$

\section{INTRODUCTION}

At present, the possibility of laser assisted charge exchange injection of a high-energy hydrogen beam with the help of powerful laser and magnetic fields is widely discussed [1-7]. A wide review on laser stripping and its achievements can be found in [2,3,7]. For this reason, in this paper we will focus mainly on the novelty and achievements of the present work.

Publications [2,7] are devoted mainly to theoretical development and design of the three-step laser-stripping scheme. The key to the scheme is the resonant excitation of hydrogen atoms by a laser. A single $\mathrm{H}^{0}$ atom can be easily excited if the laser field frequency is adjusted exactly to the resonant frequency of transition between the ground state and the upper state of the atom. However, practical excitation of the total beam is a much more complicated problem because of its angular and energy distribution. The relativistic Doppler effect in combination with the beam distribution leads to a large spread of laser frequencies near the resonance in a particle's rest frame, thus reducing the excitation efficiency. In order to overcome this difficulty, paper [2] considered adiabatic rapid passage with an approximately linearly chirped laser frequency in the particle rest frame. In practice, this was realized by tuning of the laser beam divergence.

Another approach to overcoming the Doppler spread was discussed in [5]. For high-energy beams, a strong magnetic field in the laboratory frame generates a strong electric field in the rest frame of the beam, leading to an energy broadening $\Delta E_{n}$ of Stark states. In this manner, a laser field with a Doppler spread of frequencies $\Delta \nu<$
$\Delta E_{n} / h$ should provide a good resonant excitation of the $n$th Stark level followed by instant ionization of the hydrogen atom. Using this scheme of laser stripping, there is no need to apply laser chirp. In this way the laser optics can be appreciably simplified, which is very important when using optical resonators. Moreover, calculations [7] show that the scheme in a strong magnetic field has additional advantages compared to the three-step laser-stripping scheme. The idea of paper [5] requires theory more advanced than that of paper [7] and additional calculations to justify the practical feasibility in comparison with the three-step laser-stripping scheme. It is appropriate here to mention that the direct photoionization of an atom into the continuum has a negligible "cross section" that makes the method absolutely not feasible for high efficiency stripping.

In this paper the strict quantum mechanical approach for the calculation of laser stripping via a broad shape resonance of the hydrogen atom is presented for the first time. The model is based on solving the temporal Schrödinger equation and taking into account the continuous spectrum of the broadened upper levels of the hydrogen atom in the electric field, called resonances. A quantum mechanical approach for the photoionization calculation of atomic hydrogen in an electric field has been developed in papers $[6,7]$. However, the novelty of the model presented here consists in the introduction of the continuous spectra of the broad resonances, in contrast with the discrete state model of hydrogen atom used in $[2,6,7]$. This approach significantly changes the physics of the resonance phenomena and the mathematical equations. This method, as well as $[2,6,7]$, allows us to calculate the temporal wave function 
for each atom as a function of laser and static fields $\vec{E}_{l}(t)$, $\vec{E}_{s}(t)$.

Review of fundamental works in atomic physics related to our problem reveals much work on photoionization of the hydrogen atom in an electric field. Some experimental results related to the fundamental investigation of the photoionization of hydrogen atom in electric fields were reported in [8]. Some remarkable theoretical studies [9-12] devoted to the photoionization of hydrogen atom in electric fields used a cross section or quantum perturbative approach. Strictly speaking, the cross-sectional approach in quantum mechanics has application limits. For example, it is not possible to describe the Rabi oscillations at strong laser field. Moreover, it does not allow the problem to be solved with a particular time-dependent form of the laser field.

This paper is organized as follows. In Sec. II we describe the physical model. In Sec. III we give general examples and a particular calculation for the LHC power upgrade using the design parameters of the injection beam. The examples definitely show the feasibility of laser stripping via a broad shape resonance without applying the laser chirp. The laser power remains approximately the same as in the case of the three-step laser-stripping scheme. Totally, the method of laser stripping reveals only positive features in contrast with the three-step laser-stripping scheme when considering multi-GeV beams. We use atomic units throughout this paper unless otherwise specified.

\section{PHYSICS MODEL}

Basically, the laser-stripping problem can be reduced to the problem of photoionization of atomic hydrogen by a laser field $\vec{E}_{l}(t)$ in an electric field $\vec{E}$ that takes a place in the particle rest frame after the Lorentz transformation of transverse magnetic $B_{t}$ field from the laboratory frame. Strictly speaking, the atom after the transformation will be affected by the perpendicularly crossed electric $E=$ $B_{t} v \gamma$ and magnetic $B=B_{t} \gamma$ fields where $v$ and $\gamma$ are the velocity of the beam and the relativistic factor, respectively. The estimation of the Stark-Zeeman effect in the crossed fields for a $T=4 \mathrm{GeV}$ beam and $B_{t}=2 \mathrm{~T}$ with the help of the Pauli's spinless formula for energy perturbation: $\Delta E=0.5 q \sqrt{B^{2}+9 n^{2} E^{2}}$ shows that the magnetic component $B$ gives a negligible relative perturbation $\left(<10^{-6}\right)$ of $\{0,1,0\}$ and $\{1,0,0\}$ parabolic levels compared with the electric component $E$, while the $\{0,0, \pm 1\}$ parabolic level remains unperturbed in the first order approximation. The parabolic levels are related to the frame of the electric field component $E$ directed along the $z$ axis. The choice of the 2 nd principal state of hydrogen atom and the parameters $T$ and $B_{t}$ of the laser stripping that we used for the estimation will be considered in detail in the next section. As a result of the estimation it is reasonable to omit the magnetic fields component $B$ in the particle rest frame when solving the problem.
The evolution of the hydrogen atom in the inertial rest frame of the particle can be solved with the Schrödinger equation:

$$
i \frac{\partial \Psi}{\partial t}=\left[\hat{H}_{F}+\hat{V}(t)\right] \Psi .
$$

Here $\hat{H}_{F}=\hat{H}_{0}-e z F$ is the Hamiltonian of the hydrogen atom in the pure static electric field $\vec{E}=\{0,0, F\}$ directed along the $z$ axis, $\hat{V}(t)=-e \vec{r} \vec{E}_{l}(t)$ is the interaction between the electron and the laser field $\vec{E}_{l}(t)$, and $\hat{H}_{0}$ is the Hamiltonian of unperturbed atom. For simplicity, we assume the field $\vec{E}$ to be static in the inertial rest frame of particle (1) without any circulation. Otherwise, it would be necessary to apply additional rotating transformations [7] that will formally make things more complex without any introduction of new physics. A hydrogen atom in the ground state $\Psi(\vec{r}, 0)=\psi_{0}(\vec{r})$ can be considered as the initial condition for (1).

Using the general method of solving, we can present the solution of (1) in the form of an eigenfunction expansion (see for example [13], Secs. 5, 40) of the hydrogen atom in a static electric field:

$$
\Psi(\vec{r}, t)=c_{0}(t) \psi_{0}(\vec{r})+\sum_{i} \int c_{i E}(t) \psi_{i E}(\vec{r}) d E .
$$

The integral expansion is the principal novelty made in this paper compared to the previous works. Here we suppose that the ground state $\psi_{0}(\vec{r})$ is negligibly affected by the electric field and considered as an unperturbed discrete state of the hydrogen atom. The sum in (2) is taken over the continuous spectra corresponding to the broadened parabolic states of the hydrogen atom with principal quantum number $n \geq 2$. The evolution of the wave function (1) is defined by the evolution of unknown coefficients $c_{0}(t)$ and $c_{i E}(t)$ with the initial condition $c_{0}(0)=1$.

The wave function of the ground state $\psi_{0}=$ $\exp \left[-\left(\mu^{2}+\nu^{2}\right) / 2\right] / \sqrt{\pi}$ in parabolic coordinates $x=$ $\mu \nu \cos (\varphi), y=\mu \nu \sin (\varphi), z=\left(\mu^{2}-\nu^{2}\right) / 2$ is normalized to unity. The wave functions of the continuous spectra $\psi_{i E}(\mu, \nu, \varphi)$ in the parabolic coordinates can be calculated using the method described in [14]. They can correspond to different parabolic states in the static field, called resonances. The wave function of the same resonance is normalized to the delta function (see the Appendix). The wave functions of different resonances are orthogonal to each other. The ground-state function is also orthogonal to the continuum wave functions with high precision.

We shall restrict ourselves to the practically interesting case of a moderate strength static electric field, namely, the field at which resonances are wide compared to the discrete approximation approach but do not overlap each other. A more graphic description of this case will be given at the end of the section. In this case a laser field with frequency adjusted for one particular resonance will excite a continuous spectrum only in the vicinity of this resonance, so we 
can keep only one continuum term in (2):

$$
\Psi(\vec{r}, t)=c_{0}(t) \psi_{0}(\vec{r})+\int c_{E}(t) \psi_{E}(\vec{r}) d E .
$$

The below derivations of the equations for the coefficients in (3) can be generalized without difficulty to Eq. (2).

The coefficients $c_{0}(t)$ and $c_{i E}(t)$ contain the rapidly oscillating factors $e^{-i E_{0} t}$ and $e^{-i E t}$ related to the time dependence of the stationary states. After a change of variables, $c_{0}(t)=a_{0}(t) e^{-i E_{0} t}$ and $c_{E}(t)=a_{E}(t) e^{-i E t}$, expression (3) becomes

$$
\Psi(\vec{r}, t)=a_{0}(t) \psi_{0}(\vec{r}) e^{-i E_{0} t}+\int a_{E}(t) \psi_{E}(\vec{r}) e^{-i E t} d E .
$$

Continuing with the general method of solution, we substitute (4) into (1), recalling that $\hat{H}_{F} \psi_{0}(\vec{r})=E_{0} \psi_{0}(\vec{r})$ and $\hat{H}_{F} \psi_{E}(\vec{r})=E \psi_{E}(\vec{r})$. After multiplying both sides of the equation from the left by $\psi_{0}^{*}, \psi_{E^{\prime}}^{*}$ and integrating over all space, while taking into account the orthogonality of the eigenfunctions, we obtain

$$
\begin{aligned}
i \dot{a}_{0}(t)= & \vec{E}_{l}(t) \int a_{E}(t) V_{0 E} e^{-i\left(E-E_{0}\right) t} d E \\
i \dot{a}_{E^{\prime}}(t)= & \vec{E}_{l}(t) a_{0}(t) V_{0 E^{\prime}}^{*} e^{i\left(E^{\prime}-E_{0}\right) t} \\
& +\vec{E}_{l}(t) \int a_{E}(t) V_{E^{\prime} E} e^{-i\left(E-E^{\prime}\right) t} d E .
\end{aligned}
$$

Here $V_{0 E}=\int \psi_{0}^{*} \vec{r} \psi_{E} d V$ and $V_{E^{\prime} E}=\int \psi_{E^{\prime}}^{*} \vec{r} \psi_{E} d V$ are transition dipole moments from the ground state to the continuum state and within the continuum state, respectively. It is convenient to present the rapidly oscillating laser field $\vec{E}_{l}(t)$ in the following form:

$$
\vec{E}_{l}(t)=\vec{E}_{l 0}(t) \cos [\varphi(t)]=\vec{E}_{l 0}(t) \frac{e^{i \varphi(t)}+e^{-i \varphi(t)}}{2} .
$$

As has been said above, the laser frequency is approximately adjusted to the transition frequency between the ground state and that of the resonance, i.e., $\dot{\varphi}(t) \approx E-E_{0}$. Moreover, the width of the resonance is much less than the laser frequency, i.e., $\left|E-E^{\prime}\right| \ll \dot{\varphi}(t)$. Substituting (6) into (5) and using the rotating wave approximation to cancel the rapidly oscillating terms in the exponents of (5), we obtain

$$
\begin{aligned}
i \dot{a}_{0}(t) & =\frac{\vec{E}_{l 0}(t)}{2} \int a_{E}(t) V_{0 E} e^{i \varphi(t)-i\left(E-E_{0}\right) t} d E \\
i \dot{a}_{E}(t) & =\frac{\vec{E}_{l 0}(t)}{2} a_{0}(t) V_{0 E}^{*} e^{i\left(E-E_{0}\right) t-i \varphi(t)}
\end{aligned}
$$

This is a system of integrodifferential equations for the evolution of $a_{0}(t)$ and $a_{E}(t)$ which defines the total temporal wave function (4). The evolution is characterized by the laser field $\vec{E}_{l}(t)$ and the static electric field $F$ that defines the dipole transition moments $V_{O E}$. A precise calculation of the moments can be performed using the normalized wave functions given in the Appendix. Fulfillment of the nor- malization condition $\left|a_{0}(t)\right|^{2}+\int\left|a_{E}(t)\right|^{2} d E=1$ for the wave function (5) can be shown directly from (7) indicating that

$$
\frac{d}{d t}\left[\left|a_{0}(t)\right|^{2}+\int\left|a_{E}(t)\right|^{2} d E\right]=0
$$

and bearing in mind that $a_{0}(0)=1$.

The probability of photoionization can be approximated as the probability of an electron being in the continuum state at the end of an interaction with a laser field characterized by interaction time $t_{\mathrm{int}}$ :

$$
p_{\text {ion }}=1-\left|a_{0}\left(t_{\text {int }}\right)\right|^{2} \text {. }
$$

The equations (7) and $V_{0 E}$ can be applied to nondegenerate resonances $\left\{n_{1}, n_{2}, 0\right\}$ with quantum number $m=0$. Moreover, $V_{0 E}=V_{0 E}^{*}=\left\{0,0, V_{0}\right\}$ for these states and only the projection of $\vec{E}_{l 0}(t)$ onto the $z$ axis leads to photoionization of atomic hydrogen. The resonances with $m=$ \pm 1 are doubly degenerate in energy in any static electric field and thus it is necessary to include two continuum terms in the expansion (2) with $m=+1$ and $m=-1$. The transition dipole moments from the ground state to the doubly degenerate resonance are $V_{0 E}=\left\{V_{0}, \pm i V_{0}, 0\right\}$. The new system of equations with the two degenerate resonances may be formally transformed to the system (7) using $\left|a_{E}(t)\right|^{2}=\left|a_{E}(t)_{+m}\right|^{2}+\left|a_{E}(t)_{-m}\right|^{2} \quad$ and $\vec{E}_{l 0}(t) V_{0 E}=\vec{E}_{l 0}(t) V_{0 E}^{*}=E_{l 0(x y)} V_{0} \sqrt{2}$, where $E_{l 0(x y)}$ is the projection of the laser field amplitude $\vec{E}_{l 0}(t)$ onto the $x-y$ plane. Now the doubly degenerate resonance with $m= \pm 1$ can be considered as a single resonance with $V_{0 E}=V_{0} \sqrt{2}$. Throughout this paper we will use the $V_{0 E}=V_{0} \sqrt{2}$ definition for the transition dipole moment of the degenerate resonances. For the most effective photoionization of the $\left\{n_{1}, n_{2}, \pm 1\right\}$ or $\left\{n_{1}, n_{2}, 0\right\}$ resonances, it is necessary to apply perpendicular and parallel polarization of laser to the static electric field, respectively. Throughout this paper we will consider only these polarizations of the laser field.

As an example, Fig. 1 presents the transition dipole moments $V_{0 E}$ from the ground state to the $\{0,0, \pm 1\}$ resonance as a function of electron energy $E$ for a hydrogen atom in a range of static electric fields (see details of the calculations in the Appendix).

The $\{1,0,0\}$ and $\{0,1,0\}$ resonances shown in Fig. 1 are located above and below the $\{0,0, \pm 1\}$ resonance, respectively, on the energy scale. The locations $E$ and widths $\Gamma$ of the resonances can be approximately estimated with the perturbation formulas for the Stark effect [14]. In moderately strong fields, the maxima of different resonances are located far away from each other and do not overlap. In this case, a laser field with frequency adjusted to one of the levels significantly excites energies in the nearby vicinity due to the resonance phenomena. The region of integration over energy in (7) is defined by the width of $V_{0 E}$.

From (7) it is seen that photoionization depends on the absolute value of $V_{0 E}$, which rapidly falls with increasing 


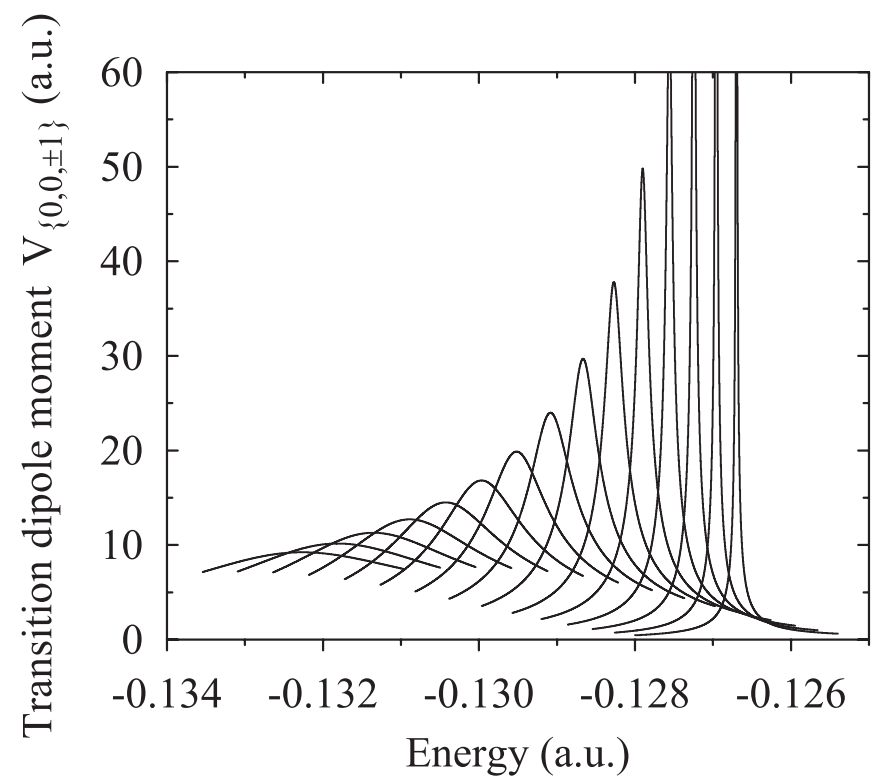

FIG. 1. The transition dipole moments $V_{0 E}$ from the ground state to the $\{0,0, \pm 1\}$ resonance as a function of electron energy for atomic hydrogen in various static electric fields. The field has the value 0.0042 a.u. for the right curve and grows with the uniform steps 0.0003 a.u. to the 0.0087 a.u. for the left curve.

static field strength (see Fig. 1). For this reason, the case of too strong electric fields is not interesting for high efficiency photoionization.

\section{EXAMPLES}

We will consider only numerical solutions of (7) in this paper. For the numerical solution, the continuous function $a_{E}(t)$ was sampled in the interval $\left[E_{R}-\Delta E, E_{R}+\Delta E\right]$ with uniform step where $E_{R}$ is the resonance energy and $V_{O E}$ is significantly small in the bounds of the interval compared with its maximum $V_{0 E_{R}}$. In this way the integrodifferential system of equations (7) is converted into a system of differential equations for $a_{0}(t), a_{E i}(t)$, where $i$ was $1, \ldots, 20000$ in our calculations. The system is then solved with the Runge-Kutta method. The computer application was configured as an external module for the PYORBIT multiphysics code [14]. The PYORBIT code has a number of accelerator physics classes designed to handle bunches. The quantum mechanical evolution of each particle in the bunch is calculated independently.

In this section we will give a few examples of photoionization calculations in static electric fields in order to demonstrate the features. The first example is devoted to a single-particle calculation. The resonance $\{0,0, \pm 1\}$ is found to be most efficient for photoionization with lowpower lasers [7]. For this reason we will use this resonance in examples below. Figure 2 presents the evolution of the ground-state population of atomic hydrogen in a variety of static electric fields. The hydrogen atom is affected by a monochromatic laser field: $\vec{E}_{l}(t)=\left\{E_{0} \cos (\omega t), 0,0\right\}$,

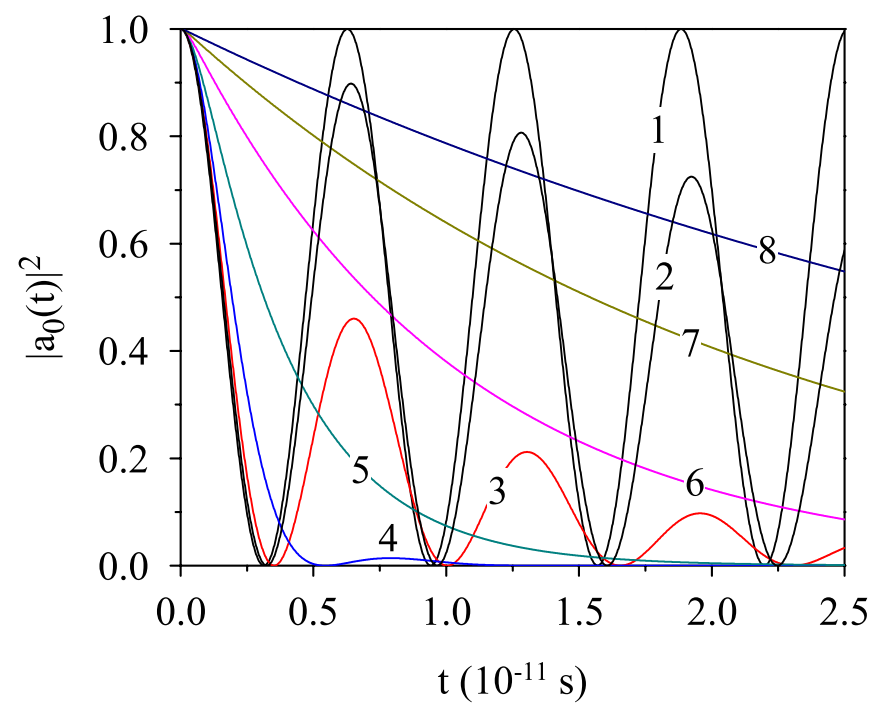

FIG. 2. (Color) Populations of the ground state of the hydrogen atom as a function of time. Curve 1 corresponds to the static field $F=0$ and curves $2-8$ correspond to static fields $0.004,0.0045$, ..., 0.007 a.u.

where $E_{0}=16.7 \mathrm{MV} / \mathrm{m}$ and the frequency $\omega$ is precisely adjusted to the resonance. Curve 1 represents pure Rabi oscillations calculated by the usual equation for the twolevel atom (see, for example, [13], Sec. 40) with transition dipole moment $\mu_{12}=128 \sqrt{2} / 243$ a.u.

It can be seen from Fig. 2 that irradiation of an unperturbed atom leads to Rabi oscillations at lower static field strengths, transforming to attenuating oscillations with increasing field strength. This fact confirms the accuracy of the derivation (A6). The stronger fields (curves 5-8 in Fig. 2) lead to a uniform decrease of the ground-state population. The application of a stronger laser field will lead to the oscillation mode of photoionization for the same static electric field. Thus, the oscillation mode of photoionization for a strong laser field or a weak static field is a quantum effect that cannot be described by the crosssectional approach [9-12].

The photoionization behavior of the hydrogen atom in different electric fields presented in Fig. 2 can be analyzed with a simplified model of an atom with two discrete levels. The equations for the evolution of the ground state and the excited upper state can be derived from the Schrödinger equation [see general formula (9) of Ref. [7] ] and have the following form [see formula (2) of Ref. [6] ]:

$$
\begin{aligned}
& \dot{a}_{0}(t)=i \frac{E_{l 0} \mu_{0 n}}{2} a_{n}(t) e^{i\left[\varphi(t)-t \Delta E_{0 n}\right]} \\
& \dot{a}_{n}(t)=i \frac{E_{l 0} \mu_{n 0}}{2} a_{0}(t) e^{-i\left[\varphi(t)-t \Delta E_{0 n}\right]}-\frac{\Gamma_{n}}{2} a_{n}(t) .
\end{aligned}
$$

Initial conditions for the equations are $a_{0}(0)=1$ and $a_{n}(0)=0$. Here $\Delta E_{0 n}$ is the energy difference between the $n$ upper state and the ground state which depends on the 
electric field because of the Stark effect. $\Gamma_{n}$ is the width of the upper level which is inversely proportional to the lifetime of the level and is responsible for the attenuation of its amplitude $a_{n}(t)$. We will consider for simplicity the frequency $\omega$ of the laser field to be always adjusted to the field dependent resonance $\Delta E_{0 n}$, i.e., $\varphi(t)-t \Delta E_{0 n}=$ $\omega t-t \Delta E_{0 n}=0$ as in the case of Fig. 2. We will also consider the dipole transition moment $\mu_{12}$ between the ground state and the $\{0,0, \pm 1\}$ state to be constant within the considered electric field range (see Fig. 3 of Ref. [7]). Equations (10) can be solved analytically. Some analysis of Eq. (10) was done in [6]. The analysis of the ground-state evolution presented in Fig. 2 can be performed via analytical solution of (10) for the ground-state amplitude:

$$
\begin{aligned}
a_{0}(t) & =e^{-\left(\Gamma_{n} / 4\right) t}\left[\frac{\Gamma_{n}}{\xi} \sinh \left(\frac{t \xi}{4}\right)+\cosh \left(\frac{t \xi}{4}\right)\right] \\
\xi & =\sqrt{\Gamma_{n}^{2}-4 E_{l 0}^{2} \mu_{0 n}^{2}} .
\end{aligned}
$$

This formula describes the curves of Fig. 2 with precision of approximately $1 \%$. In this manner it is shown that the more strict approach (7) presented in this paper agrees with the simplified one (10) very well. In the absence of the electric field, $\Gamma_{n}=0$ and the expression (11) will describe pure Rabi oscillations. For weak fields $\Gamma_{n}$ is small and the limiting expression of (11) is

$$
a_{0}(t) \approx e^{-\left(\Gamma_{n} / 4\right) t} \cos \left(\frac{t}{2} E_{l 0} \mu_{0 n}\right) .
$$

This corresponds to the damped Rabi oscillations shown in Fig. 2 for population of the ground state $\left|a_{0}(t)\right|^{2}$. Strong fields lead to a big $\Gamma_{n}$ and the limiting expression of (11) is

$$
a_{0}(t) \approx e^{-\left(E_{10}^{2} \mu_{0 n}^{2} / 2 \Gamma_{n}\right) t}
$$

which describes monotonic attenuation of the ground state. Moreover, (13) decreases more slowly for larger $\Gamma_{n}$ making depopulation of the ground state difficult. Putting $\Gamma_{n} \approx$ $2 E_{l 0} \mu_{0 n}$, we obtain the transition mode between curves 45 in Fig. 2:

$$
a_{0}(t) \approx e^{-\left(\Gamma_{n} / 4\right) t}\left(1+\frac{\Gamma_{n}}{4} t\right) .
$$

Here, it would be convenient to give the definition of the broad shape resonance as a quantum mechanical state of the hydrogen atom with the half width of the state equal to or greater than the Rabi oscillation frequency: $0.5 \Gamma_{n} \geq$ $\omega_{R}=E_{l 0} \mu_{0 n}$ or, in the case of extreme laser fields, comparable to the distance between hydrogen levels with different main quantum numbers $0.5 \Gamma_{n} \geq\left|E_{n+1}-E_{n}\right|$.

The photoionization behavior (12) and (13) can be qualitatively explained in the following way. In a weak electric field, the upper level is sharp in terms of the previous definition and behaves as the discrete level that can be effectively excited by the resonant laser. In a strong electric field the upper level is broad and cannot be excited effectively by the sharp monochromatic laser field because the latter excites only the narrow spectrum peak of width $\omega_{R}$, which corresponds to a small portion of the total broadened level. For stronger fields the width of the level increases, thereby decreasing the excited portion and making the excitation and photoionization difficult.

The transition mode (14) plays a key role for optimization of the static electric field when designing laser assisted charge exchange injection. It is considered to be close to the optimum for the photoionization efficiency of a whole beam with finite angular and energy spread. A stronger field leads to a decrease of efficiency and a weaker field leads to different off-resonant oscillations for each particle in the beam, also resulting in a decrease of efficiency. Figure 3 presents an example of optimization of a magnetic field for the LHC power upgrade [15]. The injection beam has $4 \mathrm{GeV}$ energy and other design parameters listed in [7]. The optimum magnetic field for the best ionization efficiency is found to be about $2.1 \mathrm{~T}$.

For these calculations we used an elliptical Gaussian laser beam with a wavelength $\lambda=1064 \mathrm{~nm}$, horizontal and vertical waste sizes of $w_{x}=4.0 \mathrm{~mm}, w_{y}=1.3 \mathrm{~mm}$, peak laser power $P_{0}=5 \mathrm{MW}$, and longitudinal rms duration 15 ps (see also [7] for details of the laser beam description). The photoionization of the $\mathrm{H}^{0}$ beam interaction was calculated at the laser beam waste to eliminate laser beam divergence and to provide nonchirped laser pulses in the particle rest frame. The high efficiency photoionization $p_{\text {ion }}=1-\left|a_{0}\right|^{2} \approx 0.96$ obtained from the results of Fig. 3 proves feasibility of high efficiency laser assisted charge exchange injection via the broadened Stark states [5] that is the principal result of the paper. The laser power used for this scheme is approximately the same as in the scheme of [7]. Figure 4 presents spectra of different

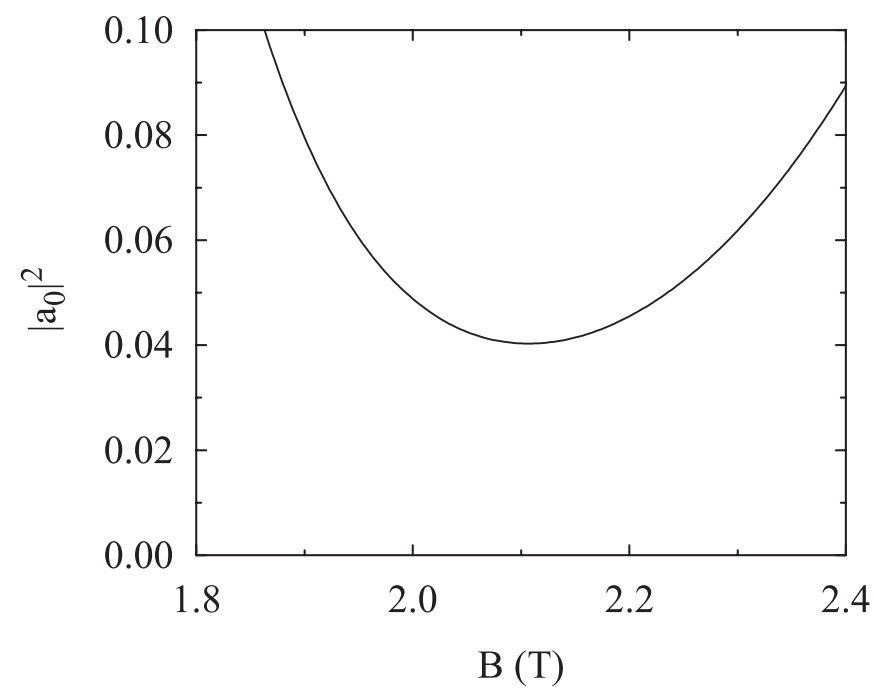

FIG. 3. Dependence of ground-state population as a function of static transverse magnetic field in the laboratory frame for a $4 \mathrm{GeV}$ beam with the design parameters listed in [7]. 


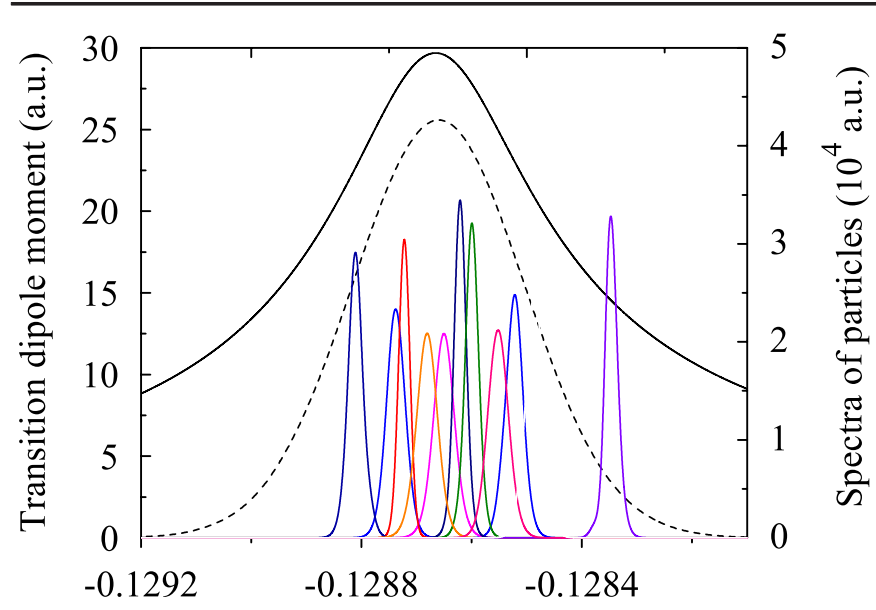

Energy (a.u.)

FIG. 4. (Color) Spectra of ten randomly chosen particles from the beam and shape of the resonance corresponding to the minimum of the curve in Fig. 3. The dashed line presents the distribution of laser frequencies in the particle rest frame conditioned by the Doppler effect.

particles of the beam excited within the broad shape resonance corresponded to the minimum of curve in Fig. 3. Different positions of the spectra are conditioned by the Doppler effect spread of laser frequency in the particle rest frame. In spite of this, most particles have a good resonance condition because of the broad shape of the resonance.

The optimal magnetic field for the $4 \mathrm{GeV}$ beam is approximately $2.1 \mathrm{~T}$ (see Fig. 3) that can be provided by permanent magnets. Less energy would require a stronger magnetic field that can be provided by the superconducting magnets making technical things more difficult.

In the end of the section it would be interesting to mention about matching of the results obtained with basically different mathematical equations (7) and (10). This fact allows us to put forward an assumption about the equivalence of the two approaches that can likely be proved mathematically. This problem together with an analytical solution of beam photoionization is the subject of future work.

\section{CONCLUSIONS}

The theory and method for precise calculation of laser stripping via a broad shape resonance of the hydrogen atom is developed. In this method we take into account for the first time both the continuous and the discrete spectrum of resonances of hydrogen atom, in comparison with previous works. The approach allows us to accurately describe the photoionization for any strength of static and laser fields. It has been shown by calculations that the scheme of laser stripping via a broad shape resonance is feasible. Moreover, it has only positive features for stripping of high-energy beams, beginning with $4 \mathrm{GeV}$, in contrast with the three-step laser-stripping scheme, as suggested in [5]. Practically, the method significantly facilitates and improves the design and optimization of parameters for laser assisted charge exchange injection.

As a next step it would be interesting to find an analytical method of solving Eq. (7) for realistic laser beams. It would be appropriate to say here that consideration of the electron spin gives additional energy splitting of atomic states according to the anomalous Zeeman effect. Estimations show that the value of the splitting is less than the $\{0,0, \pm 1\}$ resonance width by approximately a factor of 10. Nevertheless, it would be useful to take it into account in the future for more accurate calculations.

\section{ACKNOWLEDGMENTS}

This work was supported by SNS through UT-Battelle, LLC, under Contract No. DE-AC05-00OR22725 for the U.S. Department of Energy. The authors also thank S. Cousineau.

\section{APPENDIX A}

The method of calculation of continuum state functions in parabolic coordinates $x=\mu \nu \cos (\varphi), y=\mu \nu \sin (\varphi)$, $z=\left(\mu^{2}-\nu^{2}\right) / 2$ is outlined in [16]. The functions have the following form:

$$
\psi_{E}=\frac{C}{\sqrt{\mu \nu}} M(\mu) N(\nu) e^{ \pm i m \varphi},
$$

where $C$ is the normalization coefficient to be defined here. The asymptotic behavior of functions $M(\mu), N(\nu)$ is presented analytically in [16]:

$$
\begin{aligned}
& M(\mu) \underset{\mu \rightarrow \infty}{=} \frac{1}{\mu} \exp \left(-\frac{\sqrt{F}}{3} \mu^{3}+\frac{E}{\sqrt{F}} \mu\right) \\
& N(\nu) \underset{\nu \rightarrow \infty}{=} \frac{a}{\nu} \sin \left(\frac{\sqrt{F}}{3} \nu^{3}+\frac{E}{\sqrt{F}} \nu\right)+\frac{b}{\nu} \cos \left(\frac{\sqrt{F}}{3} \nu^{3}+\frac{E}{\sqrt{F}} \nu\right),
\end{aligned}
$$

with $a$ and $b$ being numerically known coefficients for given energy $E$ and electric field $F$. For normalization, it is necessary to find the coefficient $C$ in (3) to satisfy the condition

$$
\int_{V} \Psi_{E_{1}}^{*} \Psi_{E_{2}} d V=\delta\left(E_{1}-E_{2}\right)
$$

We will use a straightforward method of integration (A3) in parabolic coordinates. The volume element is $d V=$ $\mu \nu\left(\mu^{2}+\nu^{2}\right) d \mu d \nu d \varphi$. The integral (A3) is divergent at $E_{1}=E_{2}$ because of the asymptotic term $N(\nu)$. Hence, substituting the asymptotic $N(\nu)$ into (A1) and (A3) and integrating $\mu$ from 0 to $\infty$ and $\nu$ from 0 to $\infty$ and $\varphi$ from 0 to $2 \pi$, we must obtain some function $f\left(E_{1}, E_{2}\right)+\delta\left(E_{1}-\right.$ $\left.E_{2}\right)$, where the function $f$ is finite at $E_{1}=E_{2}$. Integrating (A3) with asymptotic $N(\nu)$ and separating all 
additive convergent parts for the purpose of simplification of the divergent part, we obtain the following expression:

$$
\begin{aligned}
\int_{V} \Psi_{E_{1}}^{*} \Psi_{E_{2}} d V= & f\left(E_{1}, E_{2}\right)+2 \pi C_{1} C_{2} \int_{0}^{\infty} M_{1}(\mu) M_{2}(\mu) d \mu \frac{1}{2} \int_{0}^{\infty}\left[\sin \left(\frac{\left(E_{1}-E_{2}\right) \nu}{\sqrt{F}}\right)\left(a_{1} b_{2}-a_{2} b_{1}\right)\right. \\
& \left.+\cos \left(\frac{\left(E_{1}-E_{2}\right) \nu}{\sqrt{F}}\right)\left(a_{1} a_{2}+b_{1} b_{2}\right)\right] d \nu
\end{aligned}
$$

This contains the definition of the delta function in the divergent integral. After a final transformation we obtain

$$
\begin{aligned}
\int_{V} \Psi_{E_{1}}^{*} \Psi_{E_{2}} d V= & f\left(E_{1}, E_{2}\right) \\
& +C^{2} \pi^{2} \sqrt{F}\left(a^{2}+b^{2}\right) \delta\left(E_{1}-E_{2}\right) \\
& \times \int_{0}^{\infty} M^{2}(\mu) d \mu,
\end{aligned}
$$

where $a=a_{1}=a_{2}$ and $b=b_{1}=b_{2}$. And the normalization coefficient $C$ for (A1) has the following form:

$$
C=\frac{1}{\pi \sqrt{a^{2}+b^{2}} F^{1 / 4} \sqrt{\int_{0}^{\infty} M^{2}(\mu) d \mu}} .
$$

[1] I. Yamane, Phys. Rev. ST Accel. Beams 1, 053501 (1998).

[2] V. Danilov, A. Aleksandrov, S. Assadi, S. Henderson, N. Holtkamp, T. Shea, A. Shishlo, Y. Braiman, Y. Liu, J. Barhen, and T. Zacharia, Phys. Rev. ST Accel. Beams 6, 053501 (2003).

[3] V. Danilov, A. Aleksandrov, S. Assadi, J. Barhen, W. Blokland, Y. Braiman, D. Brown, C. Deibele, W. Griece, S. Henderson, J. Holmes, Y. Liu, A. Shishlo, A. Webster, and I. N. Nesterenko, Phys. Rev. ST Accel. Beams 10, 053501 (2007).

[4] A. Aleksandrov, S. Assadi, W. Blokland, S. Cousineau, V. Danilov, C. Deibele, W. Grice, S. Henderson, J. Holmes, Y. Liu, M. Plum, A. Shishlo, A. Webster, I. N. Nesterenko, and L. Waxer, SNS TechNote 176, 2007.

[5] I. Yamane, T. Suzuki, and K. Takayama, KEK Report No. 2002-76, 2002.

[6] V. Danilov, A. Aleksandrov, S. Assadi, J. Barhen, Y.
Braiman, W. Grice, A. Shishlo, S. Henderson, B. Lang, Y. Liu, G. Murdoch, K. Potter, D. Berkaev, V. Kobets, I. Koop, V. Kuz'minykh, Yu. Shatunov, and D. Shwartz, in Proceedings of the 33rd ICFA High Intensity and High Brightness Hadron Beams, Bensheim, Germany, 2004, AIP Conf. Proc. No. 773 (AIP, New York, 2005) [http:// scitation.aip.org/getabs/servlet/GetabsServlet?prog= normal\&id=APCPCS000773000001000286000001\& idtype $=$ cvips \&gifs $=$ yes] .

[7] T. Gorlov, V. Danilov, and A. Shishlo, Phys. Rev. ST Accel. Beams 13, 050101 (2010).

[8] T. Bergeman, C. Harvey, K. B. Butterfield, H. C. Bryant, D. A. Clark, P. A. M. Gram, D. MacArthur, M. Davis, J. B. Donahue, J. Dayton, and W. W. Smith, Phys. Rev. Lett. 53, 775 (1984).

[9] G. Alvarez, R. J. Damburgand, and H. J. Silverstone, Phys. Rev. A 44, 3060 (1991).

[10] A. Alijah, J. Phys. B 25, 5043 (1992).

[11] A. Alijah and A. von Hopffgarten, J. Phys. B 27, 843 (1994).

[12] H. Suno, L. Andric, T. P. Grozdanov, and R. McCaroll, Eur. Phys. J. D 13, 213 (2001).

[13] L.D. Landau and E. M. Lifshitz, Quantum Mechanics: Non-relativistic Theory (Pergamon, Oxford, 1977).

[14] A. Shishlo, J. Holmes, and T. Gorlov, in Proceedings of the 10th International, Computational Accelerator Physics Conference (ICAP2009), San Francisco, 2009.

[15] M. Benedikt and B. Goddard, in Proceedings of the 23rd Particle Accelerator Conference, Vancouver, Canada, 2009 (IEEE, Piscataway, NJ, 2009) [http://trshare.triumf.ca/ pac09proc/Proceedings/papers/we1gri03.pdf ].

[16] R. J. Damburg and V. V. Kolosov, in Rydberg States of Atoms and Molecules, edited by R.F. Stebbings (Cambridge University Press, Cambridge, 1986). 